\title{
Hyperspectral Imaging: A New Intraoperative Tool for Pouch Assessment in Patients Undergoing Restorative Proctocolectomy
}

\author{
Boris Jansen-Winkeln ${ }^{\mathrm{a}}$ Jonathan P. Takoh ${ }^{\mathrm{a}}$ Claire Chalopin ${ }^{\mathrm{b}}$ \\ Marianne Maktabi ${ }^{b}$ Orestis Lyros ${ }^{a}$ Robert Sucher ${ }^{a} \quad$ Albrecht Hoffmeister $^{c}$ \\ Niels Teich ${ }^{d}$ Hannes Köhler $^{b}$ Ines Gockel ${ }^{a}$ \\ ${ }^{a}$ Department of Visceral, Transplant, Thoracic and Vascular Surgery, University Hospital of Leipzig, Leipzig, Germany; \\ ${ }^{b}$ Innovation Center Computer Assisted Surgery (ICCAS), University of Leipzig, Leipzig, Germany; ${ }^{\circ}$ Department \\ of Gastroenterology, University Hospital of Leipzig, Leipzig, Germany; ${ }^{d}$ Internistische Gemeinschaftspraxis für \\ Verdauungs- und Stoffwechselkrankheiten (IGVS), Leipzig and Schkeuditz, Leipzig, Germany
}

\begin{abstract}
Keywords
Hyperspectral imaging · Intraoperative imaging ·

Proctocolectomy · lleal pouch-anal anastomosis .

Anastomotic leak
\end{abstract}

\begin{abstract}
Introduction: Restorative proctocolectomy with ileal pouchanal anastomosis (IPAA) is a challenging operation. Especially the mobilization of the pouch into the pelvis can be complex. Adequate perfusion of the pouch is required for optimal healing and functioning. Methods: With hyperspectral imaging (HSI) wavelengths between 500 and 1,000 nm can be analyzed in addition to visible light and by reflecting patterns. This intraoperative procedure is non-invasive, contactfree, and no contrast medium is needed. Fifteen patients undergoing IPAA were examined prospectively, and the pouch was evaluated by HSI intraoperatively. HSI was measured in standardized fashion at 4 defined locations of the J-pouch. Each measurement took about $10 \mathrm{~s}$. The clinical postoperative course was assessed in all patients and correlated to the intraoperative HSI findings. Results: Mean near-infrared perfusion and oxygenation of patients showed values $\geq 74 \%$ for all defined pouch areas, revealing good blood supply. Three minor anastomotic leaks were detected by standard pouchoscopy in the postoperative course, which could be treated conservatively with endosponge therapy. Conclusion: $\mathrm{HSI}$ values of perfusion and oxygenation of the IPAA
\end{abstract}

were high. The leak rate is associated with redo procedures. This is reflected by the current literature and most likely related to the higher complexity of the revisional pouch operation. HSI has proved itself as a quick and effective new intraoperative tool to evaluate pouch perfusion objectively and quantitatively.

(C) 2021 The Author(s) Published by S. Karger AG, Basel

\section{Introduction}

Since its first description in 1978 by Parks and Nicholls [1], restorative proctocolectomy with ileal pouch-anal anastomosis (IPAA) has long been established as the surgical treatment of choice not only for patients with therapy-refractory ulcerative colitis (UC) and/or UC-associated (pre)neoplastic lesions, but also for patients with multiple synchronous colorectal carcinomas and familial adenomatous polyposis (FAP) [2, 3]. Furthermore, this procedure can successfully treat certain cases of colonic Crohn's disease, mainly patients without perianal and small intestinal diseases.

Indications for a 1-, 2-, or 3-stage procedure not only depend on the stage and severity of the underlying dis-

B.J.-W. and J.P.T. shared first authorship equally. H.K. and I.G. shared senior authorship equally. This study is registered under Clinicaltrials. gov: NCT03667950. mercial purposes requires written permission. 
ease, but also on the presence of sepsis, the nutritional status of the patient, the presence of comorbidities, as well as the extent of immunosuppression which has to be tapered and adjusted to the time of surgery [4-6].

Even though antidiarrheal medication is more often necessary following J-pouch reconstruction when compared to other techniques, it nevertheless still is the most frequently applied variant due to the ideal combination of technical simplicity and good long-term results [7-9].

Pouch leakage or, worse still, necrosis, leading to pelvic sepsis in the early postoperative course is associated with diminished pouch function and quality of life in the long run. With regard to this most feared complication, ideal perfusion of the distal ileal loop (via the ileocolic artery) and, of course, a tension-free anastomosis are essential [10]. Ensuring that these conditions are met often presents a compelling challenge to the surgeon especially because the mobilization of the pouch into the pelvis can be complicated by tension on the relevant vessels (the ileocolic vessels or branches of the superior mesenteric artery). Sometimes, these vessels must be dissected for length recovery. Nevertheless, adequate perfusion of the pouch still has to be guaranteed for optimal healing as a prerequisite for ideal functioning of the pouch in the postoperative course.

Pouch leaks can potentially appear at the site of the IPAA itself, at the blind ileum part, or at the side-to-side ileal-ileal anastomosis. Most frequently, insufficiencies are located at the tip of the J-pouch and lead to pelvic abscess formation, which in turn leads to sepsis in the acute stage, and in the long run to pouch-anal fistulas as well as pouch-vaginal fistulas. Data on leak rates vary in the literature. Depending on case volume of the reporting center, the surgeon's experience and specialization, as well as the type of pouch reconstruction performed, reported rates lie between 2.9 and $6.5 \%$ [8]. In their meta-analysis of short-term and long-term outcomes of J-, W- and Sileal reservoirs for restorative proctocolectomy in 4,183 patients, Lovegrove et al. [9] reported an insufficiency rate of $6.9 \%$. It is important to note that even small insufficiencies of the IPAA can lead to severe inflammation and pelvic sepsis. In a meta-analysis by Hueting et al. [11], $9.5 \%$ of patients presented with pelvic sepsis in the postoperative course due to insufficiencies or complete dehiscence of the pouch, and perineal wound infections. In patients with UC, an even accelerated risk for pelvic sepsis is postulated during the first 4 months after pouch construction as compared to FAP patients. This is attributed mainly to the immunomodulating medication and associated risks for surgical side effects [12].

Also the suture technique used seems to play a role. Authors of the Cleveland Clinic and others have proved impressively, that the J-pouch in the stapling technique without mucosectomy is associated with less morbidity,
Table 1. Patients' demographics and surgical IPAA procedures

\begin{tabular}{lc} 
Age, median (range), years & $33(18-62)$ \\
Sex, males:females, $n$ & $7: 8$ \\
Diagnosis, $n(\%)$ & \\
UC & $12(80)$ \\
FAP & $2(13)$ \\
Desmosis coli & $1(7)$ \\
Operation type, $n(\%)$ & \\
Primary J-pouch & $10(67)$ \\
$\quad$ 2-step-/3-step-approach & $3 / 7$ \\
Pouch revision/redo J-pouch & $5(33)$ \\
Stapled & $5(33)$ \\
Hand-sewn & $10(67) /$ re-do 5 \\
Laparoscopic/open surgery & $10(66) / 5(33) /$ \\
& re-do (2/3) \\
Complications, $n$ (\%) & $8(53)$ \\
None & $3(20)$ \\
Anastomotic leak (conservatively treated) & $3(20)$ \\
Diversion-pouchitis & \\
\hline
\end{tabular}

such as leaks, pouch fistulas, strictures and failure rates, when compared to hand-sewn procedures with mucosectomy $[9,13]$.

Hyperspectral imaging (HSI) is a relatively new tool for image-guided surgery and allows quantitative tissue analyses beyond the limitations of the human eye. Thus, it serves as a new intraoperative diagnostic technique in visceral surgery for optical properties of diverse tissues. In contrast to other imaging methods, HSI is contactless, non-invasive, and application of a contrast medium is not necessary. Recently, we evaluated HSI in tissue/tumor detection and classification. Also, we evaluated its use in the analyses of transection lines and gastrointestinal anastomoses during surgical procedures in order to optimize outcomes, especially to reduce anastomotic leakage [1420]. The objective of our current study was the intraoperative HSI of pouches following restorative proctocolectomy in patients with UC and FAP (primary pouches and redo cases) and to correlate the HSI findings with clinical outcomes.

\section{Materials and Methods}

The study was approved by the local Ethic Committee of the Medical Faculty of the University of Leipzig, Germany (No. 026/18-ek, January 31st, 2018). The study was conducted according to the Declaration of Helsinki. All patients have signed a written informed consent form. All patients enrolled in this trial were part of the registered Study under Clinicaltrials.gov: NCT03667950. From January 2018 to July 2019, all patients with gastrointestinal anastomoses were prospectively recorded and the consecutive patients with IPAA were selected retrospectively. Twenty-two patients received a Pouch procedure; in 7 patients, no image could be captured because the HSI camera was used in parallel. Fifteen pouches could be recorded and evaluated with the HSI camera. 

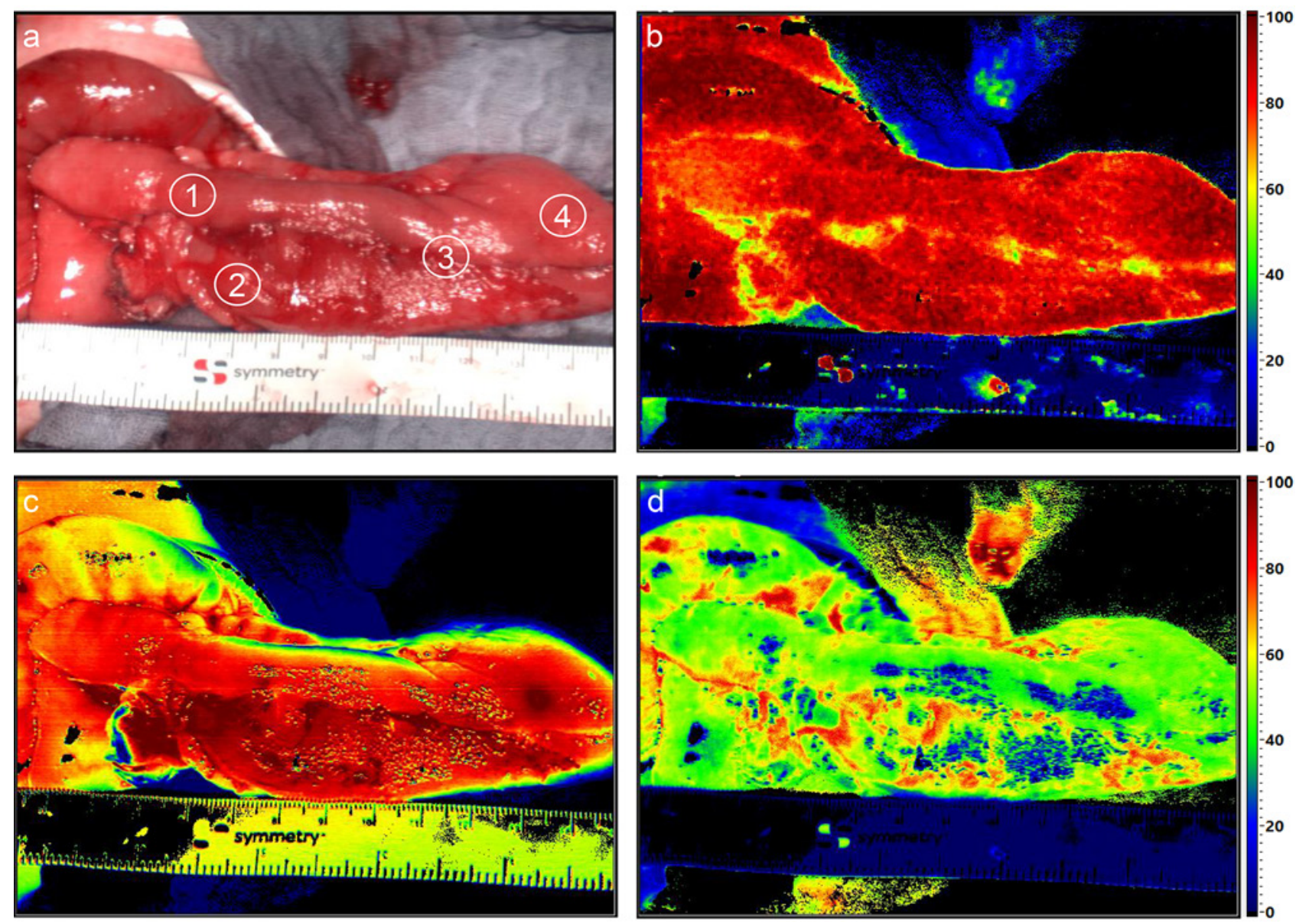

Fig. 1. a Color image of the measured J-pouch with labeled sites for (1) proximal, (2) vertex, (3) linear staple line, and (4) distal. False-color parameter images show the quantitative values for tissue oxygenation (b), near-infrared perfusion index (c), and hemoglobin content (d).

The same anesthesia protocol was used for all patients. In all patients a mean arterial pressure above $65 \mathrm{~mm} \mathrm{Hg}$ was maintained. The peripheral $\mathrm{SpO}_{2}$ prior to anastomosis was above $98 \%$ in all cases.

Our surgical technique of laparoscopic restorative proctocolectomy with IPAA is described in detail in our (video-)publication $[7,21]$. In elective patients, we routinely perform the two-stage proctocolectomy procedure with loop ileostomy at the time of IPAA, which was the most frequent variant of surgery in the described cohort of this study (Table 1). The loop ileostomy was usually reversed 6-8 weeks postoperatively. However, in patients with revisional pouch surgery, open redo J-pouch was the predominant procedure (Table 1). In brief, all J-pouches of our cohort were constructed with a volume of $100-125 \mathrm{~mL}$ and a length of $16 \mathrm{~cm}$ except for one reversal procedure in a case of pouch dysfunction and emptying problems due to dilation. In this patient, the pouch diameter was reconstructed to $12 \mathrm{~cm}$. The overall aim of all procedures was the complete removal of the substrate of the underlying colorectal pathology with simultaneous reconstruction of the gastrointestinal continuity and maintenance/re-establishment of the continence function.

As preparation technique, we chose the close rectal resection with preservation of the mesorectum and its dissection with a seal- ing device. In 2 revisional cases, the first operation had been performed in the TME (total mesocolic excision) layer, so that we again - preferred this dissection plane. About the anastomosis, the most important goal was to leave a small rectal mucosal ring above the dentate line of $1-1.5 \mathrm{~cm}$ only. Where this was well possible from the abdominal point of view, we chose to resect the rectum with a linear stapling device and then performed the circular anastomosis in double-stapling technique ( $n=5$ patients). In patients where the distance from the dentate line from could not be assessed certainly, a transanal hand suture anastomosis was carried out $(n=10 \mathrm{pa}-$ tients). All of the 5 reversal surgeries were hand sutured. In 4 male patients, either the ileocolic artery $(3 \times)$ or branches of the superior mesenteric artery $(1 \times)$ had to be dissected in order to mobilize the pouch properly and tension-free into the small pelvis.

Except for the 5 reversal surgeries, 3 colectomies were performed in a two-stage, and 7 in a three-stage approach. These 7 patients had pronounced immunosuppression and received a colectomy at first. After an average of 225 days, rectal resection was completed with pouch creation. All patients underwent structured follow-up pouchoscopies in the postoperative course at defined intervals. The follow-up was done through a telephone interview using questions designed to assess the presence of improvement in quality of life. We did not use a validated score like the SF36. This 


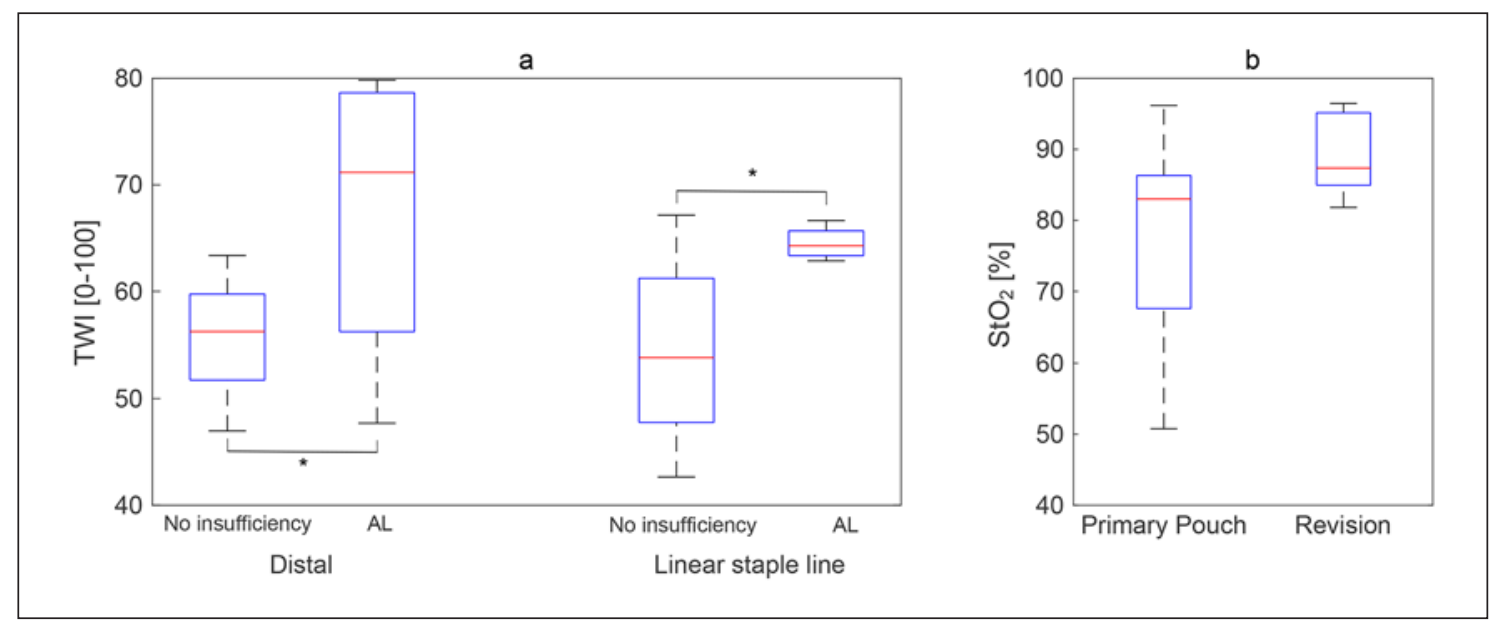

Fig. 2. a Tissue water index (TWI) at the distal and linear staple line site (ROI) for patients with and without anastomotic leaks. b Tissue oxygenation at the distal pouch site for patients with primary pouch construction and pouch revision. Data are shown as mean and SD. ${ }^{*} p<0.05$.

included assessing stool frequency, the presence of incontinence, and drawbacks in performing daily activities. Patients were asked about further operations and the subjective satisfaction with the pouch (very satisfied/satisfied/ok/dissatisfied/very dissatisfied), as well as special features. The interview took place on average after 46 weeks (6-107 weeks).

Hyperspectral images were recorded with the TIVITA ${ }^{\circledR}$ Tissue system (Diaspective Vision GmbH, Pepelow, Am Salzhaff, Germany), which provides the false-color images of physiologic tissue parameters illustrated in Figure 1. The system consists of a hyperspectral camera, which acquires data with high spectral resolution $(5 \mathrm{~nm})$ using push-broom scanning in the visible and near-infrared range $(500-1,000 \mathrm{~nm})$. The spatial resolution of the images depends on the distance to the object and the used camera lens. Spatial resolutions from 0.05 to $0.5 \mathrm{~mm}$ per pixel and resulting field of views from 13 to $550 \mathrm{~cm}^{2}$ can be achieved. The number of effective pixels of the displayed images is $640 \times 480$. The camera is surrounded by 6 halogen spots for object illumination. Camera and illumination unit are attached to a positioning arm, which is mounted on a mobile cart. Because of the HSI camera's high sensitivity to light pollution resulting in false readings, all imaging was done under a designed standard operation procedure. Prior to imaging, the camera was correctly positioned under normal lighting conditions. Using the integrated laser positioning assistant, the area of interest was placed in the plane of focus, and a test shot was taken. Once correct positioning was attained, all external light sources were switched off including the LCD monitors of computers present in the operating room. Also, all window blinds were closed. The final image was then taken under exclusive illumination by the camera's illumination unit. During imaging, the reflected light is divided into its spectral components by the spectrometer inside the hyperspectral camera. The HSI system computes 4 quantitative false-color images that represent superficial tissue oxygenation saturation $\left(\mathrm{StO}_{2}\right.$ in \%), tissue oxygenation up to 4-6 mm depth (NIR Perfusion Index [0-100]), Organ Hemoglobin Index (OHI [0-100]) and Tissue Water Index (TWI [0$100])$ at each pixel of the image. Validation, in-depth description of the parameters and their calculation were published by Holmer et al. [22].

The calculated RGB images of the recorded data were used to annotate the regions of interest (ROI) for postoperative analysis of the J-pouch. Four ROIs (proximal [PP], distal [DP], vertex, and linear staple line $[\mathrm{An}]$ ) were labeled by the operating surgeon as shown in Figure 1a. The mean value inside the ROI was calculated for each labeled site, parameter, and patient. These values were correlated with the ROI position, type of operation, and postoperative occurrence of anastomotic insufficiency.

Regarding statistical analysis, equal variances were confirmed by the $f$, test and the unpaired two-tailed Student $t$ test determined the $p$ value for statistical significance.

\section{Results}

The acquisition of hyperspectral image data was possible in all patients with neglectable prolongation of the operation procedure. HSI enabled the quantitative intraoperative visualization of J-Pouch perfusion. Patients with later anastomotic leak $(n=3)$ were indexed with "AL" and showed a significant higher tissue water content of the linear staple line ROI than patients without anastomotic leak, indexed with "NoAL" (median $\mathrm{TWI}_{\mathrm{NoAL}}=$ 54; median $\mathrm{TWI}_{\mathrm{AL}}=64 ; p=0.025$ ) and distal ROI (median $\mathrm{TWI}_{\mathrm{NoAL}}=56$; median $\mathrm{TWI}_{\mathrm{AL}}=71 ; p=0.034$ ). These patients also showed a significant higher tissue oxygenation of the distal ROI (median $\mathrm{StO}_{2} \mathrm{NoAL}=82 \%$; Median $\left.\mathrm{StO}_{2 \mathrm{AL}}=93 \% ; p=0.045\right)$. There was no significant difference of the distal ROI between patients, who underwent pouch revision compared to primary pouch proce-

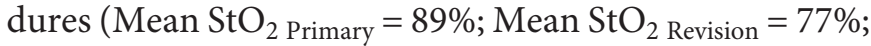
$p=0.11$ ). The value distributions are given in Figure 2 . The comparison of the ROI locations for all patients revealed significant higher tissue oxygenation of proximal (median $\mathrm{StO}_{2}=87 \% ; p=0.029$ ) and vertex (median $\left.\mathrm{StO}_{2}=88 \% ; p=0.011\right)$ sites compared to the linear staple line (median $\mathrm{StO}_{2}=79 \%$ ). Furthermore, the TWI was significantly higher at these sites (median $\mathrm{TWI}_{\mathrm{PP}}=64 \%, p=$ 0.033 ; median $\mathrm{TWI}_{\mathrm{DP}}=64 \%, p=0.027$; median $\mathrm{TWI}_{\mathrm{An}}=$ 
Fig. 3. Distribution of the region of interest mean values of tissue oxygenation - $\mathrm{StO} 2$ (a) and tissue water index (TWI; b) among the 4 pouch sites for all patients. ${ }^{*} p<0.05$.

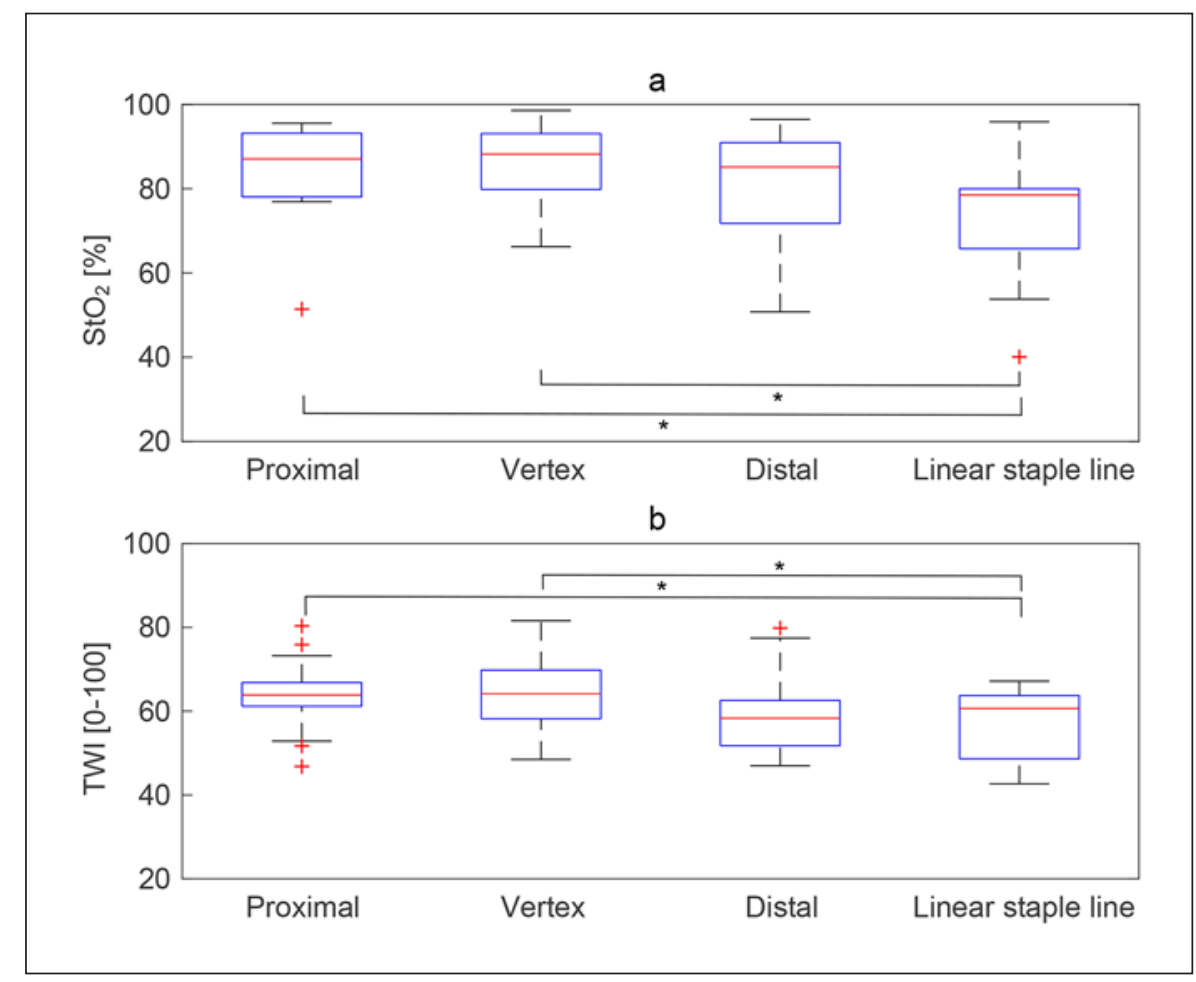

61\%). NIR Perfusion Index and OHI revealed no remarkable differences between the 4 ROI positions or patient groups. The distribution of $\mathrm{StO}_{2}$ and TWI for the different sites is given in Figure 3.

Three patients with redo surgery developed an anastomotic leak postoperatively (Table 1). The leaks were successfully treated conservatively with endosponge therapy and all healed completely. Therapy over 12-20 days was necessary in these patients. One patient had a tear of the linear stapler line over $1.5 \mathrm{~cm}$ dorsally during surgery. A primary endosponge was inserted directly and could be removed after $3 \times 4$ days. Structured pouchoscopy was performed on day 7, days 21 and 35 postoperatively, as well as before stoma reversal. Three Patients had a diversion pouchitis with purulent fluid retention. These $\mathrm{Pa}-$ tients received pouch irrigations with $\mathrm{NaCl}$-fluid at shortterm intervals, initially daily, and, depending on the clinical picture, later every 2 days to once a week. One patient with pouchitis also received Budenosid rectal foam for 1 week. This problem was eliminated in all cases after stoma reversal.

In the follow-up interview, $14 / 15$ patients confirmed they were very satisfied. Only one patient was dissatisfied after the redo procedure but experienced the situation better than before the operation. No patient needed another operation. There was no correlation between satisfaction and HSI measurement scores. The patient with dissatisfaction had redo surgery and postoperative anastomotic leakage as well as the longest operative time.

\section{Discussion}

This feasibility study is the first to our knowledge evaluating the J-pouch by HSI in patients undergoing restorative proctocolectomy for UC or FAP. Comparable data in the world literature are not available so far. Thus, our study clearly shows that HSI of the pouch following proctocolectomy is easily feasible and efficiently practicable in surgical routine. The workflow in the operating room is not prolonged or disturbed relevantly, as the intraoperative imaging takes few seconds only per recording $[16,17,19]$. Perfusion of the pouch is a key issue. Especially the mobilization of the pouch into the pelvis can be complex by tension on the relevant vessels (ileocolic vessels or branches of the superior mesenteric artery). Sometimes, these must be dissected for length recovery. Thus, in particular tissue oxygenation and perfusion HSI parameters of the IPAA could be a useful tool in the near future to predict early postoperative and long-term outcomes. However, this has to be analyzed in a large prospective-randomized trial. In our study, we found a higher median TWI and tissue oxygenation in patients with anastomosis insufficiency. The higher TWI does not come as a surprise when taking into consideration that insufficiencies in our cohort occurred exclusively in male patients having a more challenging anatomy and thus longer operation time prior to imaging. The high oxygenation level asserts an optimal perfusion of the anastomosis thus suggesting that minimizing the risk of anastomosis insufficiency entails more than optimizing perfu- 
sion. Factors such as tissue tension in the region of anastomosis play an important role in the healing process. Prediction of IPAA healing and prevention of anastomotic leaks could be a major challenge and task for intraoperative HSI in these procedures, since septic postoperative complications, mainly due to IPAA insufficiency or even necrosis, represent the highest risk for a diminished or deteriorated pouch function [23] - or, in worst case, for the necessity of pouch explantation [24]. As pouch complications and pouch function tightly correlate with patients' quality of life [25], ideal conditions of pouch parameters, as measurable by HSI, exert an outstanding impact on perfect pouch construction and technique. Our postoperative follow-up also shows that subjective dissatisfaction is related to a complicated postoperative (and intraoperative) course. We did not use a validated score. It is well known that optimum perfusion of the pouch, usually by the ileocolic artery apart from tension-free anastomosis - is a key factor for undisturbed healing. So far, this has only been judged by surgeons' subjective visualization and assessment, such as vitality signs of the intestine, pulsations of peripheral arteries, and peristalsis of the segment. New intraoperative imaging devices as HSI could be a useful instrument to objectively guide the surgeon, prevent impaired pouch perfusion and thus, serve as a major step towards enhanced patient safety. Our data clearly show that perfusion and oxygenation values were in the upper range of HSI measurements. However, normative values and borderline ranges predictive of IPAA healing have to be established yet and cannot be drawn from our preliminary data. HSI values of NIR perfusion and oxygenation of the IPAA were high in both primary and redo J-pouch procedures. Currently, there are no comparable data of alternative intraoperative pouch imaging and perfusion measurement tools, such as ICG (indocyanine green), available in the current literature.

In addition, HSI has proved itself as a quick and effective intraoperative device to evaluate pouch perfusion, without comprising or prolonging the operative procedure. In patients with primary J-pouch construction, all patients experienced an excellent short-and mid-term postoperative outcome. Due to limited follow-up, no data are available on long-term pouch survival and function in these patients. In patients with redo pouch construction of our cohort, complication rates, namely IPAA leaks, were higher in spite of similar reconstruction techniques and HSI parameters as compared to the primary group. However, this might rather be owed to the complexity of the revisional pouch procedure, and not to potentially unfavorable tissue parameters of the anastomosis, such as low perfusion and oxygenation or high water content. The higher leak rate in the redo pouch group, as also reflected by large cohorts, such as 500 patients of the Cleveland Clinic [26] is, thus, most likely not related to reduced perfusion parameters, but to the higher complexity of the revisional pouch operation. In the study of Remzi et al. [26], patients with pelvic sepsis following redo surgery were at risk developing re-failure of the revised pouch. Undoubtedly, pouch healing and functioning are multifactorial and perfusion/oxygenation, although representing major predictors, can only be viewed in the whole context of parameters beyond the tissue blood supply, such as immunosuppression, comorbidities, and acuteness of inflammation, for example, in IBD. It is well known that about $50 \%$ of patients with pouch construction develop some kind of pouchitis in the long run up to 10 years after surgery, caused by different factors, namely chronic pouch ischemia, immuno-dysregulation and disbalance of microbiota. The patterns of pouchitis and the endoscopic distribution of pouch inflammation differ widely [27]. It will be of utmost interest in future investigations to correlate distinctive intraoperative HSI constellations with the postoperative presence and extent of pouchitis in the long-term follow-up.

Another aspect that deserves discussion is the influence of parameters that might affect the assessed microcirculation. All in all, the effects of small changes in such parameters excluding extreme changes, have not yet been reliably evaluated. Because all parameters prior to HSI data acquisition were within the so-called normal range, we believe that a relevant influence can be excluded. The effect of room temperature, for example, on HSI measurement is unclear; in each of our studied patients, room temperature was static. Of course, in the postoperative phase many scenarios are conceivable in which the circulatory situation has an effect on the anastomosis, but these are extremely difficult to detect.

Regarding the HSI measurements of complete (closed) proctocolectomy specimens immediately after resection, we have initiated a study comparing these with the final histopathologic evaluation. The external HSI assessment might be helpful in order to determine the extent of resection margins intraoperatively. However, this is essential in cases with proven or suspected malignancy (e.g., in CU patients with rectal cancer and close vs. wide rectal dissection) and Crohn's disease, aiming at most possible restrictive resection extent. The "regular" cases of CU (without malignancy) and FAP, undergoing restorative proctocolectomy with well-defined, standardized surgical borders, will probably not benefit from external, serosa-sided inspection by HSI.

\section{Conclusion}

The new technique of HSI is feasible and effective in patients undergoing restorative proctocolectomy and Jpouch construction, without comprising the operative procedure. HSI-values of NIR-perfusion and oxygen- 
ation of the IPAA were high in both - primary and redo J-pouch procedures. Thus, the intraoperative strategy did not change in our cohort due to high HSI values in both groups. However, the predictive impact of intraoperative HSI is yet unclear with regard to pouch healing and pouchitis in the postoperative course. The higher leak rate in the redo pouch cohort, as already reflected by the current literature, is most likely not related to reduced perfusion parameters, but to the higher complexity of the revisional pouch operation.

\section{Statement of Ethics}

The study was approved by the local Ethic Committee of the Medical Faculty of the University of Leipzig, Germany (No. 026/18-ek, January 31, 2018). The study was conducted according to the Declaration of Helsinki. All patients have signed a written informed consent form.

\section{Conflict of Interest Statement}

All authors have no competing interests, except Hannes Koehler - he is an employee of the company "Diaspective vision," the manufacturer of the used hyperspectral camera.

\section{Funding Sources}

There is no funding.

\section{Author Contributions}

I.G. and B.J.-W. conceived and designed the study protocol. B.J.-W., I.G., C.C., and H.K. developed the methodology. B.J.-W., J.P.T., O.L., R.S., A.H., and N.T. performed the procedures and/or provided administrative, technical, or material support. H.K., I.G., B.J.-W., C.C., and M.M. analyzed and interpreted the data. I.G., B.J.-W., and J.P.T. wrote and/or revised the manuscript.

\section{References}

1 Parks AG, Nicholls RJ. Proctocolectomy without ileostomy for ulcerative colitis. BMJ. 1978 Jul;2(6130):85-8.

2 Fazio VW, Ziv Y, Church JM, Oakley JR, Lavery IC, Milsom JW, et al. Ileal pouch-anal anastomoses complications and function in 1005 patients. Ann Surg. 1995 Aug;222(2):120-7.

3 Oresland T, Fasth S, Nordgren S, Hultén L. The clinical and functional outcome after restorative proctocolectomy. A prospective study in 100 patients. Int J Colorectal Dis. 1989;4(1): 50-6.

4 Ikeuchi $\mathrm{H}$, Uchino M, Matsuoka H, Bando T, Matsumoto T, Tomita N, et al. Surgery for ulcerative colitis in 1,000 patients. Int J Colorectal Dis. 2010 Aug;25(8):959-65.

5 Travis SP, Stange EF, Lémann M, Oresland T, Bemelman WA, Chowers Y, et al.; European Crohn's and Colitis Organisation (ECCO). European evidence-based Consensus on the management of ulcerative colitis: current management. J Crohn's Colitis. 2008 Mar;2(1):2462.

6 Seifarth C, Börner L, Siegmund B, Buhr HJ, Ritz JP, Gröne J. Impact of staged surgery on quality of life in refractory ulcerative colitis. Surg Endosc. 2017 Feb;31(2):643-9.

7 Jansen-Winkeln B, Lyros O, Lachky A, Teich $\mathrm{N}$, Gockel I. [How does the ileoanal pouch keep its promises?: functioning of the ileoanal pouch after restorative proctocolectomy]. Chirurg. 2017 Dec;88(12):1033-9.

8 Sherman J, Greenstein AJ, Greenstein AJ. Ileal j pouch complications and surgical solutions: a review. Inflamm Bowel Dis. 2014 Sep;20(9): 1678-85.

9 Lovegrove RE, Heriot AG, Constantinides V, Tilney HS, Darzi AW, Fazio VW, et al. Metaanalysis of short-term and long-term outcomes of J, W and S ileal reservoirs for restorative proctocolectomy. Colorectal Dis. 2007 May;9(4):310-20.
10 Gorgun E, Remzi FH. Complications of ileoanal pouches. Clin Colon Rectal Surg. 2004 Feb;17(1):43-55.

11 Hueting WE, Buskens E, van der Tweel I, Gooszen HG, van Laarhoven CJ. Results and complications after ileal pouch anal anastomosis: a meta-analysis of 43 observational studies comprising 9,317 patients. Dig Surg. 2005;22(1-2): 69-79.

12 Heuschen U, Schmidt J, Allemeyer E, Stern J, Heuschen G. [The ileo-anal pouch procedure: Complications, quality of life, and long-term results]. Zentralbl Chir. 2001;126 Suppl 1:3642.

13 Fazio VW, Kiran RP, Remzi FH, Coffey JC, Heneghan HM, Kirat HT, et al. Ileal pouch anal anastomosis: analysis of outcome and quality of life in 3707 patients. Ann Surg. 2013 Apr; 257(4):679-85.

14 Barberio M, Longo F, Fiorillo C, et al. HYPerspectral Enhanced Reality (HYPER): a physiology-based surgical guidance tool. Surg Endosc. 2020 Apr;34(4):1736-44

15 Gockel I, Jansen-Winkeln B, Holfert N, et al. Possibilities and perspectives of hyperspectral imaging in visceral surgery. Chirurg.2020 Feb; 91(2):150-9.

16 Jansen-Winkeln B, Maktabi M, Takoh JP, Rabe SM, Barberio M, Köhler H, et al. [Hyperspectral imaging of gastrointestinal anastomoses]. Chirurg. 2018 Sep;89(9):717-25.

17 Jansen-Winkeln B, Holfert N, Köhler H, Moulla Y, Takoh JP, Rabe SM, et al. Determination of the transection margin during colorectal resection with hyperspectral imaging (HSI). Int J Colorectal Dis. 2019 Apr;34(4): 731-9.

18 Köhler H, Jansen-Winkeln B, Chalopin C, Gockel I. Hyperspectral imaging as a new optical method for the measurement of gastric conduit perfusion. Dis Esophagus. 2019 Dec; 32(10): 1 .
19 Köhler H, Jansen-Winkeln B, Maktabi M, Barberio M, Takoh J, Holfert N, et al. Evaluation of hyperspectral imaging (HSI) for the measurement of ischemic conditioning effects of the gastric conduit during esophagectomy. Surg Endosc. 2019 Nov;33(11):3775-82.

20 Maktabi M, Köhler H, Ivanova M, JansenWinkeln B, Takoh J, Niebisch S, et al. Tissue classification of oncologic esophageal resectates based on hyperspectral data. Int J CARS. 2019 Oct;14(10):1651-61.

21 Jansen-Winkeln B, Lyros O, Lachky A, Teich N, Gockel I. [Laparoscopic proctocolectomy technique : restorative proctocolectomy with ileal pouch-anal anastomosis in ulcerative colitis. Video article]. Chirurg. 2017 Sep;88(9):777-84.

22 Holmer A, Marotz J, Wahl P, Dau M, Kämmerer PW. Hyperspectral imaging in perfusion and wound diagnostics - methods and algorithms for the determination of tissue parameters. Biomed Tech (Berl). 2018 Oct;63(5):547-56.

23 Rijcken E, Senninger N, Mennigen R. [Restorative proctocolectomy for ulcerative colitis : long-term functional results and quality of life]. Chirurg. 2017 Jul;88(7):566-73.

24 Mark-Christensen A, Erichsen R, Brandsborg S, Rosenberg J, Qvist N, Thorlacius-Ussing O, et al. Long-term Risk of Cancer Following Ileal Pouch-anal Anastomosis for Ulcerative Colitis. J Crohn's Colitis. 2018 Jan;12(1):57-62.

25 McCombie A, Lee Y, Vanamala R, Gearry R, Frizelle F, McKay E, et al. Early postoperative complications have long-term impact on quality of life after restorative proctocolectomy. Medicine (Baltimore). 2016 Jul;95(27):e3966.

26 Remzi FH, Aytac E, Ashburn J, Gu J, Hull TL, Dietz DW, et al. Transabdominal Redo Ileal Pouch Surgery for Failed Restorative Proctocolectomy: Lessons Learned Over 500 Patients. Ann Surg. 2015 Oct;262(4):675-82.

27 Shen B. Problems after restorative proctocolectomy: assessment and therapy. Curr Opin Gastroenterol. 2016 Jan;32(1):49-54. 